\title{
Are random events perceived as rare? On the relationship between perceived randomness and outcome probability
}

\author{
Karl Halvor Teigen ${ }^{1} \cdot$ Gideon Keren ${ }^{2}$ \\ Published online: 3 January 2020 \\ (C) The Psychonomic Society, Inc. 2020
}

\begin{abstract}
Many daily life events, from lotteries to coincidental encounters, occur partly or entirely randomly or "by chance." Six experiments, in two different languages, explored how perceptions of randomness are related to the perceived probability of the same events - specifically, whether low-probability events were viewed as more random than similar events that were judged (rightly or wrongly) to be more likely. The experiments suggest that low-probability outcomes of stochastic events are indeed considered as being more random than medium and highly likely outcomes, even when all are produced by a "blind" (hence random) process. Degree of randomness involved in catching a bus was inversely related to the subjective probability estimates of the same event, both for correct and incorrect estimates. Unlikely coincidences were perceived to be more random than the same events presented in a more likely frame. The outcome of a match between two soccer teams was deemed to be more random when the weaker team wins than when the stronger team wins. Only extremely deviant outcomes - for instance, a top student who fails on two successive exams - made some people reject the randomness account, presumably believing that such extreme events must have a causal explanation. We conclude that people generally associate randomness with low-probability events, indicating outcomes that "cannot be predicted."
\end{abstract}

Keywords Randomness judgments $\cdot$ Subjective probabilities $\cdot$ Coincidences $\cdot$ Event perception

The concept of randomness, or chance, is formally difficult to define (Kac, 1983; Nickerson, 2002), yet it plays an important role for a number of theoretical and practical applications. From a descriptive viewpoint, laypeople also experience in their daily life numerous events that may seem to them, more informally, as partly or totally random. Such events are interchangeably described as accidental, coincidental, fortuitous, random, hazardous, or happening by chance or by "sheer luck," in contrast to those that are seen as more orderly, controllable, foreseeable, or causally determined (and thus presumably explainable). Some "chance" events are actively sought (e.g., lotteries, gambles), whereas others are rather avoided (e.g., risks, hazards); some are thought of as

Electronic supplementary material The online version of this article (https://doi.org/10.3758/s13421-019-01011-6) contains supplementary material, which is available to authorized users.

Karl Halvor Teigen

k.h.teigen@psykologi.uio.no

1 Department of Psychology, University of Oslo, Oslo, Norway

2 Tilburg University, Tilburg, The Netherlands beneficial, like serendipitous discoveries (Roberts, 1989), whereas others are seen as worrisome because of the uncertainty involved (Anderson, Carleton, Diefenbach, \& Han, 2019).

It has been argued that the role of chance in several domains is larger than most people, including social scientists, have been willing to realize (Bandura, 1982; Denrell, Fang, \& Liu, 2014; Krantz, 1998; Mlodinow, 2008; Sunstein, 2015; Taleb, 2005, 2007). It has even been claimed that "most of the things that happen to [people] happen "by accident" (Becker, 1994, p. 183). Career choices and career transitions are a case in point (Betsworth \& Hansen, 1996; Krumboltz, Foley, \& Cotter, 2013; McDonald, 2010). It has also been proposed that luck is a major factor for professional success (Frank, 2016), especially in business (Ma, 2002) and management (Parnell \& Dent, 2009), not to mention the crucial role of different varieties of good and bad luck in matters of health and disease (Fredriksen, 2005a, 2005b). Philosophers have discussed at length the many ways that "luck" permeates our lives and its potential consequences for legal and moral judgments, free will, and determinism (Church \& Hartman, 2019).

The research literature on what people consider to be a chance event has been surprisingly sparse. Past research about 
laypeople's perceptions of randomness has mostly been devoted to people's assessment of strings of events, especially random sequences of coin flips and other displays of binary events (for reviews, see Bar-Hillel \& Wagenaar, 1991; Griffiths, Daniels, Austerweil, \& Tenenbaum, 2018; Lopes, 1982; Nickerson, 2002; Oskarsson, Van Boven, McClelland, \& Hastie, 2009). In this research, people are asked either to produce sequences that supposedly could come from a random generator or to make judgments about such sequences that are presented to them. Participants in these studies are generally reported to perform poorly on both tasks, specifically exaggerating the number of alternations they believe will show up in a random pattern. Conversely, when a sequence contains long runs or displays other regularities, they think it is less likely to be random. Thus, in a series of coin flips, HTTHTHHHT is perceived to be more random than, for instance, HHHHHTTTT.

In the real world, however, people rarely encounter sequences of binary events, except for in the world of gambling. In daily life, they experience singular, unexpected events, like an accident, an unplanned meeting, a near miss, the daily ups and downs of stock prices, and ponder in such cases to which extent "randomness" has been involved in shaping outcomes. Such judgments are in turn important for ascriptions of responsibility, credit, and blame (Alicke, 2000; Gerstenberg et al., 2018), and will be used as guidelines for what we can learn from these events and how they can be prevented or promoted in the future (Loimer \& Guarneri, 1996; Zhou \& Pham, 2004).

The question examined in the present paper centres on the role of outcome probability for the perceived randomness of such events. Will rare events be judged to be more, or perhaps less, random than events that are more likely and believed to happen more often? While formal accounts do not include magnitude of probability as a criterion of randomness, informal observations suggest it might be relevant.

Probabilities are traditionally demonstrated in situations characterized by a stochastic process. Flipping an "unbiased" coin implies a probability of $1 / 2$ for heads, since both outcomes, heads and tails, are deemed equal. Similarly, the probability is $1 / 6$ for each outcome of a fair six-sided dice. Both probabilities are compatible with (and even depend upon) an assumption of randomness, but neither outcome should be regarded as more random than the other. From this line of reasoning, the probability of an outcome has no bearing upon its perceived randomness, so common and extraordinary events might be random to the same degree. What matters is the process by which they are produced rather than outcome characteristics.

There is some evidence that highly numerate individuals subscribe to a process account. In a study on people's intuitions about randomness and probability, Lecoutre, Rovira, Lecoutre, and Poitevinau (2006) found that a sample of 20 mathematicians regarded all stochastic items (coin flips, rolls of dice, and drawings from a box) in their questionnaire as random, regardless of probability and outcome symmetry, whereas "real" situations ("you will get the flu next month," or "it will rain tomorrow in Paris"), which could be explained by a variety of causes, were regarded as less random. Process definitions have also been endorsed by researchers in psychology, including Wagenaar (1988), Falk and Konold (1997), Nickerson (2002), and Oskarsson et al. (2009).

The process underlying an outcome is, however, not always open for inspection and must be assumed or imagined, partly based on outcome characteristics. For instance, people sometimes assert that an improbable event could not have occurred "by chance" because it is too far away from what could be expected from a chance generator. The same logic leads scientists to reject the null hypothesis for probabilities below an (arbitrary) level of significance, implying that such patterns of results are too distinct to occur "by chance." In a similar vein, people experiencing a "rare" or remarkable reallife event might claim that it is too unlikely to be completely coincidental. Such outcomes are alternatively attributed to some hidden mechanism like synchronicity (Inglis, 1990), conspiracies (Brotherton \& French, 2014), magic spells, or other supernatural influences. Misattributions of chance events to hidden causes are particularly common among people holding paranormal beliefs (Rogers, Qualter, \& Woods, 2016), but even without such prior beliefs, people who have survived "against all odds" may feel they have been saved through divine intervention (Teigen \& Jensen, 2011). Thus, extremely low probabilities might be used as evidence against the notion of randomness (Johansen \& Osman, 2015) and alternative mechanisms may be sought to explain the outcome.

Notwithstanding, if randomness implies a lack of order and control, we may conceive a random event as an outcome that was not expected, and hence exceptional and unlikely. This could result in an association between low probability and randomness, such that comparable outcomes will be perceived as more random if they are less likely, whereas outcomes that are expected to occur will not be judged as random.

An emphasis on low probability seems implied by authors discussing the randomness of life events (Bandura, 1982, 1998; Kimhi \& Zysberg, 2009; Krantz, 1998). Reports of randomness are typically illustrated by surprising and unusual occurrences, such as missing a plane that later crashed or meeting one's future partner by accidentally sitting next to her at a public lecture. Some philosophers have explicitly asserted that ascriptions of luck require an unlikely outcome (Baumann, 2012; Rescher, 1995). In a discussion of chance events in the life course, Shanahan and Porfeli (2006) proposed that (among some other criteria) "chance events are unlikely" (p. 100), though no evidence or arguments were offered to substantiate this claim. In interviews with students 
about their understanding of randomness, relative rarity emerged as a central characteristic in some interviews (Jolfaee, Zazkis, \& Sinclair, 2014). These authors conclude as follows: "The connection between randomness and low probability, either calculated or perceived, should be investigated further as it was not featured in prior research" (p. 405). The present studies were intended to do just that.

We propose that randomness and low probability become associated because they both imply a shortfall in predicting outcomes. We hypothesize that this connection generalizes to situations where the generation mechanism is controlled for, so that the drawing of a green ball from an urn containing only $10 \%$ green balls might appear more random than a drawing of one of the remaining $90 \%$ blue ones. The present studies were designed to test this notion, including its boundary conditions. Under which conditions will low-outcome probabilities suggest randomness, and when will they suggest the presence of nonrandom factors?

\section{Ratings of randomness}

To test the influence of outcome probabilities on perceived randomness, participants were asked to rate the degree of randomness for rare events compared with events that are not so rare within the same domain. How meaningful is such a task?

Formally, randomness can be regarded as a dichotomous construct: Either an outcome is produced by a random process, where all constituent elements are independent and have the same probability of occurring, or it is not. Yet when it comes to complex events, like binary sequences, "some events seem more random than others" (Griffiths et al., 2018, p. 85). Participants in such studies have been asked to judge degrees of randomness on a scale from completely nonrandom to completely random (e.g., Schreiber, 2014; Tyszka, Zielonka, Dacey, \& Sawitcki, 2008; Zhao, Hahn, \& Osherson, 2014; see also Table 1 in Falk \& Konold, 1997, for a review).

The emphasis of the present study is not on such prearranged or imagined sequences; rather, we examine singular events (like exam grades, traffic accidents, and the outcomes of soccer matches), that can be determined by several factors, where randomness is often thought to play a part. It is not uncommon in such instances to judge a "random component" as being small or large. Similarly, the reliability of a test can be defined according to how much the scores will vary "randomly" from one occasion to the next.

It may be difficult to know the exact meaning of ratings on a scale where randomness has not been further specified or defined. However, the notion of randomness among laypeople is presumably not very subtle and may not hinge upon a specific definition. For instance, Eagle (2018) concluded that in ordinary language people use the word "random" interchangeably with "by chance." Similarly, Teigen and Kanten
(2019) found no difference in ratings of statements containing the terms "randomly," "by chance," and "accidentally," suggesting that people's usages of these terms are roughly equivalent. Thus, despite the lack of a precise notion of how randomness should be defined, people seem to think there is a class of disorderly occurrences that are not foreseeable, difficult to manage, and give a proper causal explanation. They might also have beliefs about to which degree such "random factors" are involved in producing a specific outcome.

\section{The present research}

Six experiments were designed to examine the relationship between perceived randomness and probability judgments, specifically testing the low-probability account. For this purpose, participants rated the randomness of events that were comparable in terms of process, but where the outcome probabilities differed, objectively (Experiments 1 and 2) or subjectively assessed (Experiments 3 and 4). In all these studies, the causal factors could be assumed to be roughly the same both for high-probability and low-probability outcomes.

Experiments 5 and 6 were added to explore the boundary conditions for the link between low probability and perceived randomness, which had been observed in the preceding studies. This was done by describing outcomes that were even more extreme and hence less likely than in the other studies. We included scenarios where alternative explanations of the unlikely events (soccer and exam results) could be imagined.

In all studies, participants were asked to rate the perceived degree of randomness involved in producing a particular outcome, ranging from not at all to completely random. Three experiments $(2,4$, and 5$)$ were conducted in Norwegian, with the term "tilfeldig," which typically is translated with random, by accident, or by chance (Haugen, 1984). In two of the experiments (2 and 6), participants were also invited to explain their ratings.

\section{Experiment 1: Blind draws}

In this study, a high-probability outcome was compared with a low-probability outcome both occurring as a result of a random process - namely, marbles blindly drawn from an urn containing 10 green and 90 blue marbles. Participants were told about one player (Karl) who could win by drawing blindly a green marble from the urn. Another player (John) participated in a similar lottery where he could win the same amount by drawing one of the blue marbles. Both players won. The question was whether these two outcomes would be regarded as equally random, or whether the low-probability outcome would be judged as less random or more random than the high-probability outcome. Two related data collections, 
Experiment 1a and Experiment 1b, were performed to control for the generality of the results.

\section{Method}

For Experiment 1a, we recruited 240 Amazon Mechanical Turk workers, randomly assigned to three conditions. Participants in the joint condition were told about both players and simply asked whose win (of \$500) was more random. Participants in two separate conditions were told either about Karl or about John. In these conditions, participants were asked to assess the randomness of Karl's [John's] winning on a rating scale from 1 (totally not random) to 7 (totally random).

In Experiment 1b, 285 MTurk workers were randomly allocated to four conditions in a $2 \times 2$ design, with magnitude of prize ( $\$ 10$ vs. $\$ 1,000$ ), and winning once versus winning three times in a row, as two independent factors. Participants in all conditions had a choice between three response alternatives: Karl's winning is more random, John's winning is more random, and Karl's and John's winnings are equally random.

\section{Results}

Experiment 1a In the joint condition, 73 of 79 participants (92.4\%) selected Karl's winning as being more random than John's $(p<.001$ by binomial test), supporting the hypothesis of low probabilities being more random. However, there was in this condition no "equal" option, preventing participants to base their judgments on the random process (which indicates that Karl and John's results are equally random since they are both drawn "blindly").

In the separate conditions, Karl's low-probability win was judged as significantly more random compared with John's high-probability win $\left(M_{\text {Karl }}=5.85, S D=1.31\right.$ vs. $M_{\text {John }}=$ 4.33, $S D=1.85), t(158)=5.96, p<.0001, d=0.94$. These ratings indicate that not all "blind draws" are regarded as being completely random. However, there could still be a subset of participants who thought both outcomes were equally random.

Experiment 1b When given three response options, $47 \%$ of the participants chose to say that both winners won equally randomly. Among the remaining subjects, most favoured Karl as being the most random (43\%), whereas only a small minority (10\%) thought that John was more random. Similar distributions of answers were obtained in all conditions (see Fig. 1). To simplify analysis, answers were coded as -1 (John), 0 (Equal), and 1(Karl). Means were 0.36, 0.39, 0.15, and 0.44 for Conditions $1-4$, respectively, and all means were significantly different from zero. A $2 \times 2$ ANOVA did not show effect of prize magnitude, $F(1,281)=1.04, p=.31$, but a weak effect of winning once versus thrice, $F(1,281)=4.44$, $p=.036$, due to the slightly deviant distribution in Condition 3 .

\section{Discussion}

Both experiments described a stochastic situation where according to a process criterion one would expect people to find all outcomes, regardless of probability, as equally random. Yet this option, even when explicitly presented, was not universally endorsed. Those who did not answer equal (in Experiment $1 \mathrm{~b}$ ), or were not given such an option (in Experiment 1a), judged unlikely outcomes to be much more random than the likely ones. This judgment persisted even for probabilities as low as .001 (winning $\$ 1,000$ with $p=.1$ three times in a row).

\section{Experiment 2: Candidate selection by throw of dice}

This study was designed to compare perceived randomness based on throws of dice, with one, three, or five alternative winning possibilities. In an exploratory study, MTurk workers $(N=242)$ were randomly allocated to three conditions and asked to imagine that a firm was searching for candidates to fill one [three] [five] managerial position(s). The search committee ended up with a short list of six equally qualified nominees and used dice for making their final choice. John was one of the six shortlisted candidates and was subsequently chosen by the dice as [one of] the winning candidate(s). Participants in this study estimated John's probability of being chosen on a scale of zero to 100 and rated randomness on a 1 to 7 scale, yielding higher randomness scores in the lowprobability condition (with only one position to be filled) than in the two other conditions $\left(M_{1}=5.66, S D=1.43\right.$ vs. $M_{3}=$ 4.94, $S D=1.62$ and $\left.M_{5}=4.94, S D=1.66\right), F(2,239)=5.516$, $p=.004$, by a one-way ANOVA. However, many participants found outcome probabilities to be difficult to assess. As a more transparent test of the perceived association between low probability and randomness, participants in the following experiment received three levels of outcome probability to be considered jointly, and assessed on a rating scale instead of with percentages. ${ }^{1}$ We expected that participants would correctly perceive the probabilities of winning as increasing with the number of "favourable" outcomes and, compatible with the previous experiment, judge the outcome with the highest probability to be least random.

\footnotetext{
${ }^{1}$ This experiment was based on a suggestion by Maya Bar-Hillel.
} 


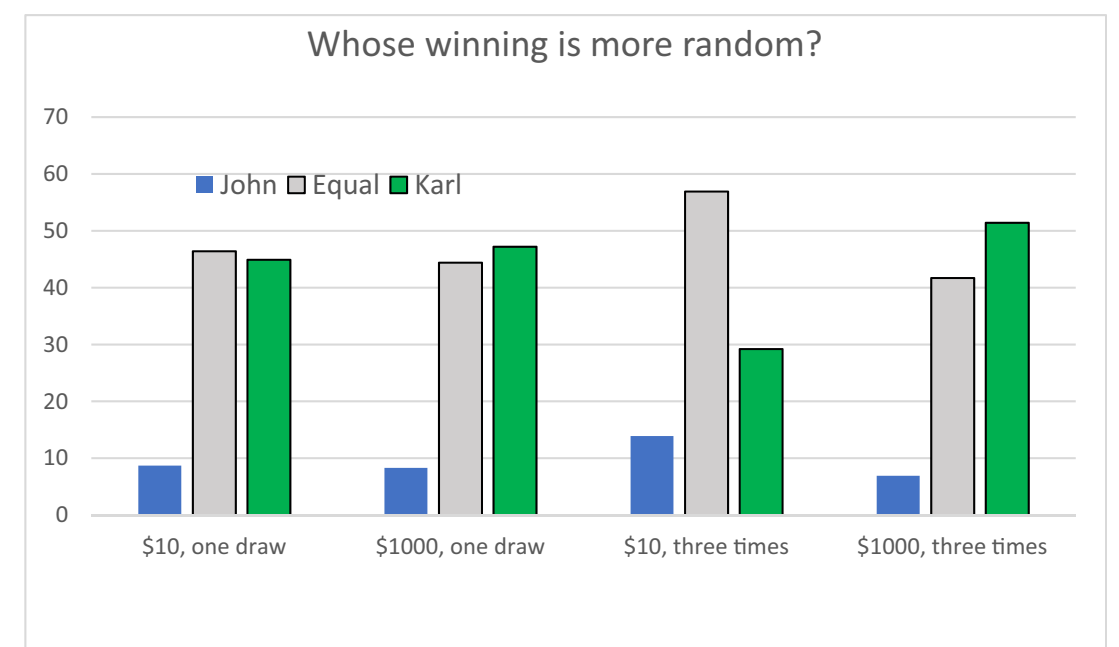

Fig. 1 Percentages of participants that describe a high-probability winner (John) or a low-probability winner (Karl) as more random, in four conditions, Experiment $1 \mathrm{~b}$

\section{Method}

Participants were 133 students at a Norwegian university (65\% women, median age 20 years). Two responses were discarded because of inconsistent answers (the ratings were in conflict with their own explanations of the same ratings). All received a brief scenario about a producer of games of dice who thought it was a good idea to select job applicants to their company by rolling dice. They were then told about three applicants: Ted, who would be offered a job if he got a 2, 3, 4,5 , or 6 on his dice; Tim, who would get an offer if he got 4 , 5 , or 6 , and Tom only if he got a 6 on the dice. How likely would it be for each of them to receive a job offer? Degrees of probability were rated on a scale from 1 (very low probability) to 7 (very high probability).

Participants were then told that the dice throws led to job offers for all three applicants, and were asked: "How random is in your opinion this outcome for each applicant?" Degrees of randomness were to be rated on a scale from 1 (not at all random) to 7 (totally random). Finally, all participants were asked to briefly explain why they had rated randomness the way they did.

\section{Results}

Mean ratings of probability and randomness are shown in Fig. 2. Probability ratings were, as expected, high for Ted and low for Tom, with Tim in between. A one-way ANOVA for repeated measures shows a highly significant effect, $F(2,264)=848.74, p<.0001, \eta_{\mathrm{p}}{ }^{2}=.865$. Randomness ratings follow the opposite pattern, $F(2$, $258)=120.63, p<.0001, \eta_{\mathrm{p}}{ }^{2}=.483$. A closer inspection of the data shows that 47 participants $(36.2 \%)$ regarded all the outcomes as equally random. Of these, 44 gave top ratings (7) to all of them, whereas three rated them as not at all random (since everything, in their opinion, is determined). Unequal ratings were generally explained by explicit references to the probabilities involved. Likely outcomes are not random; the least likely will be most random. Some claimed that this was logical, whereas others said they used their intuition or just guessed. Those who asserted that all outcomes were equally random argued that all dice throws are simply random regardless of probabilities and outcomes. Some experienced a dilemma between alternative answers: "Dice throws are actually always random, but to rate all these outcomes 7 would feel odd."

\section{Experiment 3: Catching the bus}

Experiment 3 was designed to examine the relationship between probabilities and chance in a context outside of the field of lotteries and gambling, where probabilities can be derived from observed frequencies.

As a cover story, we used a situation from Teigen and Keren (2007) about Fred, who has recorded the frequencies of accurate and delayed bus arrivals at his local bus stop. He concludes that his bus to work arrives before schedule in 1 out of 10 cases, is $0-10$ minutes late in 8 of 10 cases, and is more than 10 minutes delayed in one case out of 10 . This distribution indicates that Fred will have a $90 \%$ chance of catching the bus if he comes to the bus stop on time, and a $10 \%$ chance if he comes 10 min late. In the original study, participants were asked about his chances of catching the bus if he arrives on time and waits for 10 minutes without seeing a bus. This question is surprisingly difficult; most participants conclude either that his chances have not changed (still 90\%) or that they are now down to $10 \%$. Very few realize that his chances are now $50 \%$, as we can rule out the most likely event (the bus did not come in the $0-10 \mathrm{~min}$ interval), and are left with two 


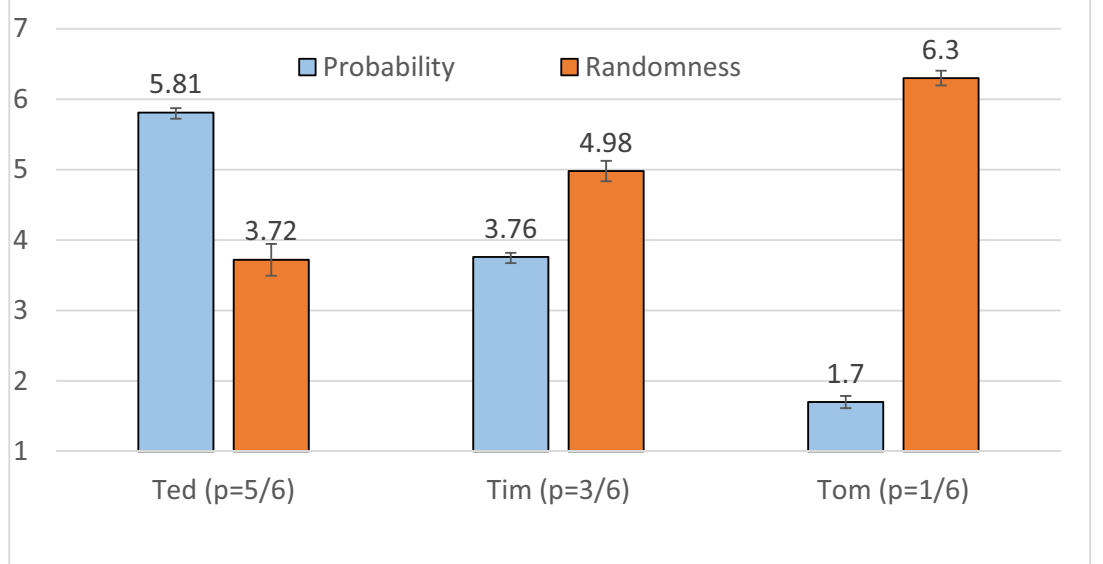

Fig. 2 Mean ratings of probability of being offered a job by throwing dice, and mean ratings of randomness of a successful outcome, Experiment 2. (Error bars represent \pm SEM)

equally infrequent alternatives (that the bus came too early or that it has still not arrived). Participants in Experiment 3 were also asked simpler questions about Fred's chances to catch the bus if he arrived at the bus stop at varying points in time (with no mention made about his waiting time).

\section{Method}

Participants were 242 American MTurk workers who received this task appended to an unrelated questionnaire; $47.5 \%$ women, mean age $=37.5$ years $(S D=11.2)$. They were randomly allocated to four conditions, receiving different information about Fred's arrival at the bus stop.

All questionnaires contained the vignette about Fred (based on Teigen \& Keren, 2007), who in Condition A arrives at the bus stop exactly on time, is 5 minutes late in Condition B, and 10 minutes late in Condition C. In Condition D he comes on time and waits for $10 \mathrm{~min}$ without the bus arriving.

In all conditions, participants were asked (1) What is the probability (chance) that the bus will still arrive? Give a number between 0 and $100 \%$. (2) Suppose that Fred actually caught the bus that day. To what extent will you describe this as a random event? Rated 1 (not at all random) to 7 (totally random).

\section{Results}

Participants in Conditions A and C succeeded reasonably well in estimating Fred's probabilities, with correct modal estimates of $10 \%$ and $90 \%$ chance. Median and mean probability estimates are displayed in the left panel of Table 1. For Condition B, no exact probability can be computed, as the distribution of departures within the 0-10 min interval was not specified. Most participants in this condition agreed that he would have between $10 \%$ and $90 \%$ chance, but with more probability estimates above than below $50 \%$.
Replicating Teigen and Keren (2007), estimates in Condition D formed a bimodal distribution, with 30 participants $(49 \%)$ giving low probabilities (in the $0 \%-20 \%$ range), and $20(33 \%)$ producing high probabilities (in the $80 \%-100 \%$ range). Only five participants $(8 \%)$ produced the correct estimate-namely, $50 \%$.

Randomness ratings differed between conditions, as shown in Table 1. A comparison of Conditions A-C shows that Fred's success in catching the bus is considered more random when he arrives late than when he arrives on time, and more random when he is 10 minutes late than when he is only 5 minutes late. A one-way ANOVA of randomness ratings results in a highly significant main effect of condition, $F(2,178)=10.712, p<.001$, $\eta_{\mathrm{p}}{ }^{2}=.107$. Pairwise comparisons show that all three conditions are significantly different from each other: A-B: $t(119)=2.48$, $p=.015$; B-C: $t(118)=2.11, p=.037$; A-C: $t(119)=4.11$, $p<.001$. Catching the bus after 10 minutes waiting is, as predicted, less random than catching it after 10 minutes without waiting, D-C: $t(119)=3.01, p=.003$.

An inverse relationship between probability estimate and randomness can also be demonstrated within each condition, as shown by the negative correlations in the last column of Table 1 . The correlations indicate that participants who (rightly or wrongly) estimated Fred's chances of catching the bus as low tended to think of this event as more random, whereas those who thought his chances were high regarded this event as less random, or not random at all. These results are compatible with the low-probability account, in a context where probabilities are based on frequencies rather than random draws.

\section{Experiment 4: Coincidences}

A coincidence, for instance an accident involving two parties, can sometimes be regarded as more or less likely depending 
Table 1 Probability estimates and randomness ratings for four different scenarios of Fred waiting for the bus, Experiment 3

\begin{tabular}{llllll}
\hline Conditions & \multicolumn{2}{l}{ Probability estimates } & $N$ & Randomness ratings (1-7) & Correlations (within each condition) \\
\cline { 2 - 5 } & Median & Mean $(S D)$ & & Mean (SD) & $r_{\text {probabilities/randomness }}$ \\
\hline A 7:00 & $90 \%$ & $71.8(30.4)$ & 61 & $2.49(1.74)$ & $-.69^{* *}$ \\
B 7:05 & $65 \%$ & $59.6(30.4)$ & 60 & $3.32(1.92)$ & $-.35^{* *}$ \\
C 7:10 & $10 \%$ & $27.5(26.7)$ & 60 & $4.05(1.90)$ & $-.34^{* *}$ \\
D 7:00-7:10 & $25 \%$ & $44.1(38.4)$ & 61 & $2.98(2.00)$ & $-.35^{* *}$ \\
\hline
\end{tabular}

${ }^{\mathrm{a}}$ The distribution is bimodal, with $49.2 \%$ low probability estimates (in the $0 \%-20 \%$ range), and $38.8 \%$ high probability estimates (in the $80 \%-100 \%$ range). $* * p<.01$

upon the way it is described. Imagine a drunk driver involved in a collision with a truck. When the accident is described as a story about a drunk driver, the collision is not very surprising. If the driver is drunk enough, we will expect him sooner or later to crash his car. From the perspective of the truck driver, who has no previous record of accidents, the happening may appear more unlikely. It may also appear as more random, as an event occurring to him "out of the blue." In a pilot study, MTurk workers $(N=160)$ rated this event on a 1-7 scale. When this accident was framed as a story about a (sober) truck driver involved in a crash with a drunk driver, it was rated much more random $(M=4.32)$ than when told, in another condition, as a story about a drunk driver crashing with the truck $(M=3.27)$, the difference being highly significant, $t(158)=3.30, p=.001$. Participants in this study were not asked to evaluate the probabilities for the event, thus preventing the calculations of the relationship between randomness and probabilities.

The present study was conducted to replicate this finding with a broader selection of scenario-based events that might look more or less "coincidental" (and more or less likely) depending upon perspective. Employing a between-group design, the events were rated either for randomness, or for probability. In line with the low-probability account, we expected the subjectively less likely versions of the stories to be also rated as more random. To make randomness and probability ratings more comparable, probabilities were in this study performed on a 1-7 rating scale instead of as $0 \%-100 \%$ estimates.

\section{Method}

Participants were 178 students attending an introductory course in psychology at the University of Tromsø, Norway (76\% women, median age 20 years). They received a short questionnaire containing four vignettes of events that were framed to appear more or less likely depending on perspective. The first vignette was adapted from Fox and Rottenstreich's (2003) demonstration of different probabilities assigned to class-based and case-based partitions of the same outcome space. Participants in their experiments estimated about $15 \%$ chance for "the hottest day next week will be Sunday" (class-based condition), but assigned a median estimate of 30\% to the same event, when framed as the probability that "Sunday will be hotter than any other day next week" (case-based condition). The other three vignettes described incidents that were constructed to look more likely, and perhaps less coincidental, from the perspective of one narrator/ actor than from that of another: A fatal car accident involving a drunk driver and a reliable truck driver; a coincidental meeting between a famous author and one of his readers; and a teacher selecting exam questions that happen to match the only chapters read by a poorly prepared student. All these events were designed to appear more special or "unique" from one perspective than from another. For instance, it was assumed that a coincidental encounter between a reader and his favourite author would appear less remarkable for the author (who has already met some of his readers) than for the reader (who never met an author before). Similarly, a teacher can expect that his questions will occasionally match the knowledge of a poorly prepared student, whereas the poorly prepared student cannot expect the teacher to select matching questions. The vignettes framed to appear less probable were labelled A, whereas the versions framed to appear more plausible were labelled B. For a full translation of the vignettes, see Appendix.

Participants were randomly allocated to four conditions in a $2 \times 2$ design, with story version (A vs. B) as one factor and response scale (randomness vs. probability) as the second factor. Participants in the randomness conditions were asked to judge the A or B version of all stories for randomness on a 7point scale. Participants in the probability conditions received the same stories, but rated them according to how likely they appeared on a 7-point scale from 1 (extremely unlikely) to 7 (extremely likely).

\section{Results}

Overall, the vignettes were viewed as describing not very likely situations $\left(M_{\text {probability }}=3.72\right)$, with a considerable 
amount of randomness involved $\left(M_{\text {randomness }}=5.22\right)$. Notably, the most likely situation (the car crash) was seen as the least random, whereas the two most random situations (the coincidental meeting of a reader and an author on the plane, and exam questions matching the only chapters read by a poorly prepared student) were at the same time perceived as the least likely (see Table 2). More important, the A versions of the vignettes proved to be judged less likely than the $\mathrm{B}$ versions; $t(88)=3.32, p=.001, d=0.70$. They were at the same time rated as more random, in line with the low-probability account, $t(89)=2.72, p=.008, d=0.48$. A $2 \times 2$ ANOVA of the mean score portrayed in Fig. 3 revealed a highly significant interaction between frame and rating scale $F(1,177)=$ $17.90, p<.001, \eta_{\mathrm{p}}^{2}=.092$. An inspection of ratings presented in Table 2 showed this pattern to be replicated with significant interactions $(p<.05)$ for all four individual vignettes.

\section{Discussion}

Vignette 1 replicated Fox and Rottenstreich's (2003) finding that a case-based description makes a hot Sunday appear quite likely (it can be hot or not so hot) compared with a class-based description where Sunday is presented as one among the other (7) days in the week. This difference emerged in a context where only ratings were called for and was not dependent on calculations of odds or percentages. The asymmetry between the parties involved in the other vignettes might make one version of the event appear more "unique" and special, and hence more unlikely than the other. Regardless of the mechanisms involved, events that appeared more likely were also seen as less random, and vice versa, for a variety of singular events.

\section{Experiment 5: Outcomes of soccer matches}

Experiment 5 was set up to test a boundary condition for the low probability account by introducing unexpected outcomes of soccer matches. People, including experts (Hughes \& Franks, 2005), consider soccer matches to involve quite a bit

Table 2 Mean ratings (standard deviations in parentheses) of probability and randomness (1-7) in A and B versions of four singular events, Experiment 4

\begin{tabular}{llllll}
\hline Vignette & Probability & & & \multicolumn{2}{l}{ Randomness } \\
\cline { 2 - 3 } \cline { 6 - 6 } \cline { 6 - 6 } & A version & B version & & A version & B version \\
\hline Hot Sunday & $3.53(1.55)$ & $3.89(1.43)$ & & $5.77(1.88)$ & $4.35(2.22)$ \\
Car accident & $5.00(1.58)$ & $5.69(1.46)$ & & $4.06(1.55)$ & $3.63(2.20)$ \\
Meeting & $2.38(1.53)$ & $3.20(1.59)$ & & $6.10(1.29)$ & $5.95(1.46)$ \\
Exam question & $2.73(1.57)$ & $3.38(1.68)$ & & $6.04(0.94)$ & $5.72(1.48)$ \\
\hline
\end{tabular}

of chance occurrences in addition to the teams' more stable skilled qualities. ${ }^{2}$ We assumed that an expected outcome, like a strong team beating a weak one, will be judged as less dependent on chance than the outcome of a match between two more equal teams, where the results could go both ways. A very unexpected outcome (as when a weak team beats a strong team) might be more ambiguous. On one hand, such a result could be produced by a unique combination of lucky circumstances favouring the weaker team; on the other hand, the result may be so unusual that people would reject the notion of chance altogether and search for a causal explanation.

\section{Method}

Two related data collections were performed. In Study 5a, students at a Norwegian university college $(N=96)$ received questionnaires entitled either Probabilities in Soccer or Randomness in Soccer. After rating their own interest in soccer (on a 1-5 scale), they were told about three teams with standings at the top of the English Premier League, three teams that were located at the bottom, and four medium teams drawn from the middle of the table. All participants were asked to imagine four matches where one team came out as winner: ${ }^{3}$

1. Chelsea (strong team) wins the match against Hull (weak team)

2. Middlesbrough (weak team) wins against Tottenham (strong team)

3. Stoke (medium team) wins against Southampton (another medium team)

4. Sunderland (weak team) wins 5-0 against Manchester City (strong team)

Participants in the probability condition were asked to estimate the likelihood of these results, as percentages between $0 \%$ and $100 \%$. Participants in the randomness condition were instead asked to rate to which extent the result could have been due to randomness (chance factors) on a 1-7 scale.

Study $5 \mathrm{~b}$ was run as a replication of the randomness condition with a new and larger sample of students $(N=151)$, who were attending an introductory lecture at a different Norwegian university (the data collections took place with less than 1 week's interval, and were based on statistics from the English Premier League during the previous season). The second data collection was designed to replicate an intriguing

\footnotetext{
${ }^{2}$ In addition, football (soccer) supporters distinguish between a factor they call "luck" and pure randomness (Wagenaar \& Keren, 1988).

${ }^{3}$ A fifth match resulting in a draw (involving two medium teams) was included in Study 5a as a filler item, but not repeated in Study 5b. This outcome was estimated to be equally probable $(50.2 \%)$ and with a similar a randomness as the winning of one of the medium teams
} 


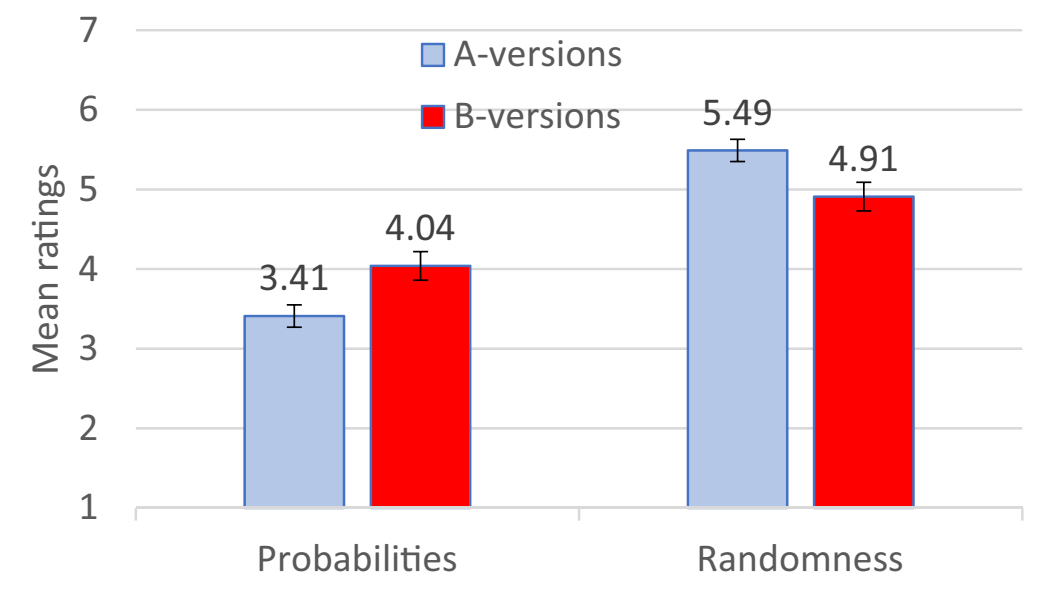

Fig. 3 Mean ratings of probability and randomness of A and B versions of singular events, all vignettes combined, Experiment 4. (Error bars represent \pm SEM)

pattern of result from the randomness condition in Study 5a, as described below. The probability condition was omitted from the replication study, as the estimates obtained in Study 5a were unambiguous.

\section{Results}

Mean estimates in the probability condition of Experiment 5a are presented in the left column of Table 3. They show, as expected, that participants considered a strong team's win over a weaker one as very likely (modal estimates of 70\%$80 \%$ ), and correspondingly that it was quite unlikely (modal estimates of 20\%-30\%) that a weak team would beat a much stronger one. It was especially unlikely that an underdog would beat a top team with a crushing 5-0 victory (modal estimates 10\%-20\%). Further, and in line with previous studies, when a strong team wins, participants believed that there was very little randomness involved. When a medium team wins, more randomness might be implied. Mean randomness scores for the two unlikely outcomes were of a similar magnitude. However, a closer inspection of the individual ratings revealed an important difference, as one half of the participants assumed that 5-0 must be very much due to chance, whereas the other half denied that chance alone could account for such an extreme outcome, leading to a bimodal distribution of randomness ratings for this particular match. This was tested by converting randomness scores into absolute deviations from the mean. Mean absolute deviations (MAD) were higher for the two matches where the weak team won, as shown in Table 3. This was especially the case in the 5-0 match, which participants believed was either very random or not random at all.

In the replication study (Experiment $5 b$ ), a majority believed that the two weak team victories were more random than nonrandom. Again, not all participants agreed on this issue, leading to large deviations around the mean, significantly higher for the 5-0 match than for the other, unspecified weak team victory $\left(\mathrm{MAD}_{5-0}=1.57\right.$ vs. $\mathrm{MAD}_{\text {unspecified }}=$

Table 3 Mean probability estimates and randomness scores (with mean absolute deviations [MAD]) for expected and surprising outcomes of a soccer match, Experiment 5

\begin{tabular}{|c|c|c|c|c|c|}
\hline \multirow[t]{2}{*}{ Outcome of match } & \multirow{2}{*}{$\begin{array}{l}\text { Probability } \\
\text { Study 5a } \\
\text { Mean estimate }\end{array}$} & \multicolumn{2}{|l|}{$\begin{array}{l}\text { Randomness } \\
\text { Study } 5 \mathrm{a}\end{array}$} & \multicolumn{2}{|l|}{$\begin{array}{l}\text { Randomness } \\
\text { Study } 5 \mathrm{~b}\end{array}$} \\
\hline & & Mean score & MAD & Mean score & MAD \\
\hline Strong team wins & $70.5 \%$ a & $2.08 \mathrm{a}$ & $0.63 \mathrm{a}$ & $2.04 \mathrm{a}$ & $0.55_{\mathrm{a}}$ \\
\hline Medium team wins & $51.5 \% \mathrm{~b}$ & $4.04_{b}$ & $0.95_{b}$ & $4.17 \mathrm{~b}$ & $1.24_{\mathrm{c}}$ \\
\hline Weak team wins & $35.9 \% \mathrm{c}$ & $4.17 \mathrm{~b}$ & $1.35 \mathrm{c}$ & $4.70_{c}$ & $1.01_{\mathrm{b}}$ \\
\hline Weak team wins $5-0$ & $23.8 \% \mathrm{~d}$ & $4.04_{b}$ & $1.66_{\mathrm{d}}$ & $4.78_{c}$ & $1.57 \mathrm{~d}$ \\
\hline
\end{tabular}

Note. Numbers with different subscripts in each column are significantly different from each other $(p<.001$ for mean estimates and mean scores, $p<.05$ for MAD) 
$1.01), t(150)=6.73, p<.001$. This pattern of results suggests that extremely unexpected outcomes (at least in soccer) can be accounted for in two rather different ways: one by sheer randomness; alternatively, when the result is perceived as extremely unlikely, there is a tendency to search for a potential causal factor that might have intervened. As remarked by one participant after the experiment: "The fact that the low-ranked team won 5-0 means that their play, this day, must have been excellent. The outcome was accordingly not random but must have been determined by their superior performance."

The current results differed from the preceding studies by indicating that a low-probability event, for some participants, could be viewed as reflecting less randomness. The present participants did not claim expertise in soccer, as their selfrated interest scores were rather low $(M=2.17$ and 2.31 in Experiments $5 \mathrm{a}$ and $5 \mathrm{~b}$, respectively), but a closer inspection of data revealed that both participants with minimal interest and participants with an above-average interest for soccer were split on this issue.

\section{Experiment 6: Extreme outcomes}

The soccer experiment suggests that an extreme result might lead some participants to reject the randomness hypothesis and regard a very unlikely event as nonrandom. Presumably, such extreme results arouse curiosity and beg for a search of an explanation. Experiment 6 tested this conjecture in two domains that differ in their a priori probability of randomness: exam results, which are meant to reflect the candidates' proficiency rather than their luck, versus drawings from an urn, which are supposed to be completely random except in the unlikely case of fraud or magic.

Imagine you are asked to predict a student's future exam grades. The student has a good record, earning mostly $A$ s in the past. Such a pattern of grades will evoke expectations about similar grades to be likely in the future, and will, by most observers, not be perceived as random. However, we know that exam grades are not entirely reliable, but are bound to fluctuate due to variations in the candidate's preparedness, fatigue, mood, examiner's preferences, uses of the grade scale, and so on. Such factors would make an occasional "random" grade of $B$ appear in the school report of an otherwise excellent student. Even a more unlikely $C$ might be attributed to a conjunction of unfortunate circumstances ruining her exam. But imagine that the student fails, perhaps not only once, but twice! Such outcomes are so deviant that randomness may not sound as a sufficient account. Experiment 6a was set up to test this conjecture.

Experiment $6 \mathrm{~b}$ was designed to replicate the lottery from Experiment 1 with an even more unlikely outcome (one player with low odds wins twice). Here, the null hypothesis (random draws) has a high prior probability but might perhaps be discarded if the results are too deviant.

\section{Method}

Participants were 213 Mechanical Turk workers who were randomly allocated to three conditions (two conditions in Experiment 6a and one condition in Experiment 6b). Four questionnaires were discarded as incomplete.

Experiment 6a Questionnaires given to participants described briefly two students, Ann and Carol, who in their first year of college had obtained a series of excellent grades, followed by two poorer ones. For Ann, the first five grades were $A, B+, A$, $A^{-}$, and $A^{-}$. On her next two exams she received one $C$ and one $B-$. Carol had obtained a similar series, $A, A-, A, B+$, and $A$, before failing her next two exams.

Participants in the randomness condition were asked, for each of the two students, to assess (on a 1-7 scale) the extent they considered the two most recent exam results to be random. They were also asked to briefly explain why (open-ended question). Participants in the probability condition rated the probability of the two most recent exam results on 7-point scales and asked to explain their ratings, as above.

Experiment 6b Participants in this experiment received a lottery questionnaire where the first question was similar to the one used in Experiment 1a about a player who draws a green marble from an urn with a minority of green (winning) and a majority of blue (not winning) marbles. The text differed from the original by increasing the prize to $\$ 2,000$ and reducing the winning probabilities to 5 out of 100 marbles. In addition, the word "blindly" was removed from the description of the drawing. The win was rated for randomness on a 7-point scale, and explanations were solicited, as in Experiment 6a.

Next, they were told that the same player entered the lottery for the second time, and won again. How random was the draw (1-7), and why was it considered random or not random?

In this experiment questions about likelihood were omitted, as probabilities could be inferred from the composition of the urn, and two wins in a row are obviously much less probable than just one.

\section{Results}

Participants evaluating the exam scenario generally believed that exam grades are not random, as they are intended to measure the student's knowledge and skills. Thus, the ratings of both students were mostly in the lower half of the scale, as indicated by the mean randomness ratings shown in Table 4 . Very few regarded the variations in Ann's grade as due to random fluctuations. She received accordingly low 
Table 4 Mean probability estimates and randomness scores (with mean absolute deviations [MAD]) for poor exam grades and lottery wins, Experiments $6 \mathrm{a}$ and $6 \mathrm{~b}$

\begin{tabular}{llll}
\hline Scenario & Probability (1-7) & \multicolumn{2}{l}{ Randomness (1-7) } \\
\cline { 3 - 4 } & Mean score & Mean score & MAD \\
\hline Exam scenario (6a) & & & \\
Two poorer grades (Ann) & $3.34_{\mathrm{a}}$ & $3.16_{\mathrm{a}}$ & $1.36_{\mathrm{a}}$ \\
Two failed grades (Carol) & $1.93_{\mathrm{b}}$ & $3.24_{\mathrm{a}}$ & $2.02_{\mathrm{b}}$ \\
Lottery scenario (6b) & Objective probability & & \\
One win & $1 / 20$ & $6.29_{\mathrm{b}}$ & $0.88_{\mathrm{c}}$ \\
Two wins & $1 / 400$ & $6.11_{\mathrm{b}}$ & $1.06_{\mathrm{d}}$ \\
\hline
\end{tabular}

Note. Numbers with different subscripts in each column are significantly different from each other $(p<.001$ for Experiment $6 \mathrm{a}$ and $p<.05$ for Experiment 6b)

randomness scores that were, on average, similar to those associated with the much more dramatic change in Carol's grades. However, Carol's grades were viewed as much more unlikely, $t(66)=7.90, p<.001$, suggesting in this experiment a dissociation between randomness and probability. A closer inspection of the ratings show that participants disagreed more about the randomness of Carol's grades than those of Ann, as reflected by a significantly higher MAD score, $t(67)=4.26, p<.001$. Although mean randomness scores were about the same, less than a third $(28.4 \%)$ thought both results were equally random. A slightly greater number $(31.3 \%)$ judged Carol's grades to be more random than the grades of Ann, whereas $40.3 \%$ thought they were less random.

Again, extreme outcomes seemed to have caused a split between those who think that such results cannot be due to chance and those who think that they indicate an even greater random component (as predicted by the low-probability account), leading to a bimodal distribution of ratings. For Ann, the most frequent randomness scores were 2 and 5, for Carol, they were 1 (not at all random), 2 and 7 (totally random). This split is reflected in the explanations given, which showed either a strong tendency to invent potential causal explanations for what might have happened ("she didn't study"; "she has developed a problem"), or simply emphasizing the anomaly of failure by an otherwise good student ("not typical of her"; "these seem like strong deviations"). Explanations of the first kind were associated with low-randomness scores, whereas explanations of the second kind were typical for highrandomness ratings. Of those who held Carol's result to be less random than Ann's, a majority (75\%) suggested possible reasons or just asserted that something must have happened: "there must be a reason." In contrast, only five participants out of 21 (24\%) suggested reasons for why Carol's results were more random. The others just pointed out that it was unusual or anomalous for her, as she was otherwise a good student.
The difference in type of explanations between these two groups is highly significant, $\chi^{2}(1,49)=12.63, p<.001$.

The lottery scenario was, as expected, regarded as extremely random, and remained so even after the player was reported to win for the second time. Most participants did not change their randomness ratings, explaining that "the situation had not changed." Yet there were voices of disagreement leading to higher MAD scores after the player had won twice, $t(69)=$ $2.44, p<.017$. A few participants lowered their randomness rating, commenting that two wins in a row were "a little suspicious," whereas others held that two wins were more random than just one, as "the odds are even lower." Randomness ratings were, however, already close to the ceiling after the first draw, limiting the possibility of still higher scores.

\section{General discussion}

The present set of studies constitutes the first attempt to examine empirically how perceived randomness of singular events is related to their judged probability. We reviewed arguments for different, seemingly plausible accounts for such a relationship, but subsequent experiments offered most consistent support for the low-probability account: Low-probability events will be perceived as more random than comparable outcomes that are estimated (rightly or wrongly) to have a higher probability of occurrence. This supports the more informal observations by Shanahan and Porfeli (2006) and Jolfaee et al. (2014), and is congruent with the fact that real-life stories about random happenings, particularly coincidences, are typically illustrated by descriptions of low-probability events (Bandura, 1982; Johansen \& Osman, 2015).

The results of the first four experiments all supported the low-probability hypothesis rather than a process account, endorsed by scientists who think that randomness "objectively" depends upon the generating mechanism (Fitelson \& Osherson, 2015; Lecoutre et al., 2006; Nickerson, 2002). Experiment 5, which compared probability estimates and perceived randomness of selected soccer results, indicated a potential boundary condition for this rule. Results that were considered highly likely were again perceived as being less random than those that were not considered so likely, whereas exceptionally extreme unlikely results turned out to be ambiguous, as participants were split into those who thought that such results indicated a very high degree of randomness and those who thought that such results could not be due to chance. This split was replicated in Experiment 6, where very deviant exam grades were viewed as being either more random (because they were unusual) or less random (because there had to be a reason). However, for low-probability events to appear nonrandom a nonrandom explanation has to be available. Thus, unexpected grades (Experiment 6a) are easier 
perceived as nonrandom than unexpected lottery results (Experiment 6b).

Comparing our results with randomness judgments performed within the binary sequence paradigm, a striking contrast can be observed. In these studies patterns were judged less random when they were perceived as unlikely outputs from a series of tosses by a coin. In Falk and Konold's (1997) studies, ratings of "apparent randomness" were in fact obtained by asking participants about the likelihood of achieving particular sequences randomly, and then concluding that unlikely outcomes meant not random. Our studies indicate the opposite-namely, that for singular events in daily life, unlikely indicates more random. These differences suggest that studies of binary sequences have limitations as a universal model of the perception of randomness (see also Matthews, 2013, on the evaluation of streaks of different kinds).

These evaluations are not as incompatible as they may seem. Judgments of randomness in sequences assume the existence of a random generator producing inchoate strings, where order is "surprising" (Feldman, 2004) and anomalous. Participants in these tasks make judgments based on an assumption of disorder, where observations of irregularity are expected and, in a way, considered as default. Outside of this rather artificial universe, however, people will look for, and expect, some degree of predictability and order. They will find irregular events to be the exception rather than the rule, and only draw conclusions about randomness in the "unlikely" case of aberrant events that cannot be predicted. By this epistemic attitude they manage to preserve a model of the world as basically orderly and explainable.

In the present studies, we have compared participants' solutions to a task of predicting outcomes (probability estimates) with a task of postdicting hypotheses (randomness ratings). In terms of conditional probabilities, this changed their task from considering $p($ data $\mid \mathrm{H})$ to expressing their opinions about the inverse relationship $p(\mathrm{H} \mid$ Data). To do so in a meaningful way, one needs to have an idea not only about $p($ data $\mid \mathrm{H})$, but also about the prior probability of potential alternative hypotheses, and how compatible the actual outcomes are with both alternative hypotheses, as required by Bayes's theorem. It is reasonable to assume that a search for alternative hypotheses will emerge when $p($ data $\mid \mathrm{H})$ is quite low, unless the process is so well described that the role of other contributing causes can be controlled for. To illustrate, the winner of the lottery in Experiment 1a had only $10 \%$ chance, yet the fact that he won could not rule out randomness, as the process of a blind draw did not allow the winning to be explained in any other way. This was replicated in Experiments $1 \mathrm{~b}$ and $6 \mathrm{~b}$ with even lower probabilities. In contrast, the factors responsible for the outcome of a soccer match include situational determinants and skills, making it easier to produce a narrative that highlights factors different from randomness. So, when an extraordinary and unlikely 5-0 outcome happens, some participants decided to look for potential reasons that made this result appear more plausible, and hence less random than before. These apparently divergent judgments do not invalidate the link between low probability and high perceived randomness, as they may simply stem from an attempt to think that extraordinary outcomes must be due to an overriding cause, making them more likely (and less random) than originally assumed. A similar process can be observed when exceptional coincidences are "explained" by recourse to magical or supernatural forces. They then become more likely (and not random) by means of an unlikely (magical) theory of coincidences (Griffiths \& Tenenbaum, 2007).

We do not claim that all low-probability events will be regarded as random, and certainly not that probability is the only determinant for attributing an outcome to randomness, or chance. Other potential determinants of randomness could be examined by manipulating other variables - for instance, causal factors, skill, intentionality, and effort. Our results indicate that low probabilities constitute one (supposedly important) facet in the perception of randomness, though we do not claim that it is the only one.

Why should low $p$ events be regarded as more random? Low $p$ events need not be uncaused or unintentional, and even quite infrequent happenings (like Halley's comet appearing once in 75 years) can form a pattern. We propose that the answer may reside in two related characteristics of subjective randomness. People consider an event as random if it appears as disconnected from the general flow of events, like an unmotivated cough in the middle of a sentence or an unexpected computer crash before the document is saved. In these cases, the "random" events cannot be conceived as causally related to the main story line. It is also in the nature of such events to be unforeseeable. They are deviant and form exceptions to the rule. By this logic, it was less foreseeable (and more random) that Karl in Experiment 1 drew a winning marble from his $10 \%$ urn than John did, as John's urn contained $90 \%$ winning marbles.

Indeed, unpredictability has been suggested as an optional definition of randomness - for instance, as independence (zero autocorrelations) between the parts of a random sequence, where one part cannot be predicted from preceding parts (Neuringer, 1986; Nickerson, 2002). Also, in the case of blind draws from an urn with unequal frequency of different colors, all marbles (but not all colors) have the same probability of being chosen, implying unpredictability on the level of individual marbles (but not on the level of colors). Our participants appear to go one step further and reason that degree of randomness depends on outcome features: a drawing of the dominant color is less random, by being more expected than other colors.

The present studies did not instruct participants as to what should be meant by a random or a nonrandom outcome. This was done deliberately, in order to avoid directing our respondents towards a specific interpretation of the term. Like 
probability, randomness is a polysemous concept (Hertwig \& Gigerenzer, 1999), having multiple, related meanings. Accordingly, we do not claim that this term was used by all participants in exactly the same way. Notwithstanding, the results showed a remarkable consistency of low-probability events being rated as being more random across a wide range of situations with speakers of two different languages.

The idea that random events are rare can have several important implications. One is exaggerated beliefs in foreseeability and control, as indicated by research on the hindsight bias (Roese \& Vohs, 2012). It might reinforce a preference for intentional rather than accidental explanations of behaviour as demonstrated by studies of the "intentionality bias" (Reich, Kupor \& Smith, 2018; Rosset, 2008). It may lead historians, social scientists, and psychologists to underestimate the role of randomness in shaping individual and collective history (Krantz, 1998; Sunstein, 2015), and make them look for patterns, plans, and explanations behind phenomena that cannot be adequately attributed to single causes or to a purposeful design.

An association between randomness and low probability may suggest that random events are in themselves insignificant and do not, as a rule, give rise to important changes. As John Stuart Mill (1856) observed, people tend to think that effects share important characteristics with their causes; large effects are supposed to have large causes, and evil effects are supposed to flow from evil forces (Nisbett \& Ross, 1980). By a similar logic, one may believe that random and unlikely events can be taken lightly, if they have only in their power to produce slight and insignificant consequences. What people think about the "magnitude" of random events and their potential causal power has been recently been examined by Teigen and Kanten (2019). Their perceived low probability suggests that they can be easily ignored or discounted as "exceptions." Correspondingly, one rarely stops to consider whether frequent or more noteworthy events could be due to chance.

Open practices statement Data files for all experiments are submitted as electronic supplementary material. None of the experiments were preregistered. Material to Experiment 4 is included in the appendix. Materials for the other experiments are available upon request.

\section{Appendix}

Material used in Experiment 4 (English translations)

Many large or small events in life appear to happen randomly or "by coincidence." We describe below four incidents where random factors are involved. [Randomness condition: In your opinion, to what extent can each of these events be considered random?] [Probability condition: Some of them appear to be more or less likely than others. In your opinion, to what extent can each of these events be considered likely?]
1

A: In Oslo, the highest temperature of the week occurred on Sunday.

B: In Oslo, the temperature on Sunday was higher than the other days of the week.

2

A: Paul, a truck driver with no previous record of accidents, collides head-on with a very drunk driver, John, driving in the opposite direction. The truck driver escapes from the collision uninjured, whereas the drunk driver is killed in the crash.

$\mathrm{B}$ : John is very drunk when driving his car and collides headon with a large truck driving in the opposite direction. The truck driver, Paul, who has no previous record of accidents, escapes from the collision uninjured, whereas the drunk driver is killed in the crash.

3

A: A student named Oscar is travelling from Oslo to London. He is a great fan of Jo Nesbø, a famous Norwegian writer of crime novels, and is reading his latest book. He discovers that the guy seated next to him in the plane is actually Jo Nesbø himself. Now he can ask him to sign the book.

B: Jo Nesbø, a famous Norwegian writer of crime novels, is traveling from Oslo to London. He discovers that the guy seated next to him in the plane, a student named Oscar, is actually reading his latest book. Oscar is a great fan of Nesbø and can now ask him to sign the book.

4

A: Ruth is a student who sits for an exam in modern history. She has only read the first two chapters in the history book and expects to fail. The teacher usually selects questions from all parts of the book, but this time all his questions are from Chapters 1 and 2. Ruth is very happy with the teacher's choice. Now she will pass the exam without any problems.

B: A teacher prepares questions for an exam in modern history. He usually selects questions from all parts of the history book, but this time all his questions are from Chapters 1 and 2. One student, Ruth, is very happy with the teacher's choice. She has only read the two first chapters in the book and expects to fail. Now she will pass the exam without any problems.

\section{References}

Alicke, M. D. (2000). Culpable control and the psychology of blame. Psychological Bulletin, 126, 556-574. https://doi.org/10.1037/ 0033-2909.126.4.556

Anderson, E. C., Carleton, R. N., Diefenbach, M., \& Han, P. K. J. (2019). The relationship between uncertainty and affect. Frontiers in Psychology, 10(2504). https://doi.org/10.3389/fpsyg.2019.02504 
Bandura, A. (1982). The psychology of chance encounters and life paths. American Psychologist, 37, 747-755. https://doi.org/10.1037/0003066X.37.7.747

Bandura, A. (1998). Exploration of fortuitous determinants of life paths. Psychological Inquiry, 9, 95-99. https://doi.org/10.1207/ s15327965pli0902_2

Bar-Hillel, M., \& Wagenaar, W. A. (1991). The perception of randomness. Advances in Applied Mathematics, 12, 428-454. https://doi. org/10.1016/0196-8858(91)90029-I

Baumann, P. (2012). No luck with knowledge? On a dogma of epistemology. Philosophy and Phenomenological Research, 89(3), 523551. https://doi.org/10.1111/j.1933-1592.2012.00622.x

Becker, H. S. (1994). "Foi Por Acaso": Conceptualizing coincidence. The Sociological Quarterly, 35, 183-194. https://doi.org/10.1111/j. 1533-8525.1994.tb00406.x

Betsworth, D. G., \& Hanson, J. C. (1996). The categorization of serendipitous career development events. Journal of Career Assessment, 4(1), 91-98. https://doi.org/10.1177/106907279600400106

Brotherton, R., \& French, C. C. (2014). Belief in conspiracy theories and susceptibility to the conjunction fallacy. Applied Cognitive Psychology, 28, 238-248. https://doi.org/10.1002/acp.2995

Church, I. M., \& Hartman, R. J. (Eds.). (2019). Routledge handbook of the philosophy and psychology of luck. London, England: Routledge.

Denrell, J., Fang, C., \& Liu, C. (2014). Perspective: Chance explanations in the management sciences. Organization Science, 26(3), 923-940. https://doi.org/10.1287/orsc.2014.0946

Eagle, A. (2018). Chance versus randomness. In E. N. Zalta (Ed.), The Stanford encyclopedia of philosophy. Retrieved from https://plato. stanford.edu/archives/spr2018/entries/chance-randomness/

Falk, R., \& Konold, C. (1997). Making sense of randomness: Implicit encoding as a basis for judgment. Psychological Review, 104(2), 301-318. https://doi.org/10.1037/0033-295X.104.2.301

Feldman, J. (2004). How surprising is a simple pattern? Quantifying "Eureka!" Cognition, 93, 199-224. https://doi.org/10.1016/j. cognition.2003.09.013

Fitelson, B., \& Osherson, D. (2015). Remarks on "random sequences." Australasian Journal of Logic, 12(1), 11-16.

Fox, C. R., \& Rottenstreich, Y. (2003). Partition priming in judgment under uncertainty. Psychological Science, 13, 195-200. https://doi. org/10.1111/1467-9280.02431

Frank, R. H. (2016). Success and luck: Good fortune and the myth of meritocracy. Princeton NJ: Princeton University Press.

Fredriksen, S. (2005a). Bad luck and the tragedy of modern medicine. Oslo, Norway: Faculty of Medicine, University of Oslo.

Fredriksen, S. (2005b). Luck, risk, and blame. Journal of Medicine and Philosophy, 30, 535-553. https://doi.org/10.1080/ 03605310500253105

Gerstenberg, T., Ullman, T. D., Nagel, J., Kleiman-Weiner, M.., Lagnado, D. A., \& Tenenbaum, J. B. (2018). Lucky or clever? From expectations to responsibility judgments. Cognition, 177, 122-141 https:// doi.org/10.1016/j.cognition.2018.03.019

Griffiths, T. L., Daniels, D., Austerweil, J. L., \& Tenenbaum, J. B. (2018). Subjective randomness as statistical inference. Cognitive Psychology, 103, 85-109. https://doi.org/10.1016/j.cogpsych.2018. 02.003

Griffiths, T. L., \& Tenenbaum, J. B. (2007). From mere coincidences to meaningful discoveries. Cognition, 103, 180-226. https://doi.org/ 10.1016/j.cognition.2006.03.004

Haugen, E. (1984). Norwegian-English dictionary. Oslo, Norway: Universitetsforlaget.

Hertwig, R., \& Gigerenzer, G. (1999). The 'conjunction fallacy' revisited: How intelligent inferences look like reasoning errors. Journal of Behavioral Decision Making, 12, 275-306. https://doi.org/10. 1002/(SICI)1099-0771(199912)12:4<275::AID-BDM323>3.0.CO; $2-$
Inglis, B. (1990). Coincidence: A matter of chance-Or synchronicity? London, England: Hutchinson.

Johansen, M. K., \& Osman, M. (2015). Coincidences: A fundamental consequence of rational cognition. New Ideas in Psychology, 39, 34 44. https://doi.org/10.1016/j.newideapsych.2015.07.001

Jolfaee, S., Zazkis, R, \& Sinclair, N. (2014).“It is very, very random because it doesn't happen very often": Examining learners' discourse on randomness. In E. J. Cernoff \& B. Sriraman (Eds.), Probabilistic thinking (pp. 397-416). Dordrecht, Netherlands: Springer. https://doi.org/10.1007/978-94-007-7155-0_21

Kac, M. (1983). Marginalia: What is random? American Scientist, 71, 405-406.

Kimhi, S., \& Zysberg, L. (2009). How people understand their world: Perceived randomness of rare life events. The Journal of Psychology, 143(5), 521-532. https://doi.org/10.3200/JRL.143.5. 521-532

Krantz, D. L. (1998). Taming chance: Social science and everyday narratives. Psychological Inquiry, 9, 87-94. https://doi.org/10.1207/ s15327965pli0902 1

Krumboltz, J. D., Foley, P. F., \& Cotter, E. W. (2013). Applying the happenstance learning theory to involuntary career transitions. The Career Development Quarterly, 61, 15-26. https://doi.org/10.1002/ j.2161-0045.2013.00032.x

Lecoutre, M.-P., Rovira, K., Lecoutre, B., \& Poitevineau, J. (2006). People's intuitions about randomness and probability: An empirical study. Statistics Education Research Journal, 5, 20-35.

Loimer, H., \& Guarnieri, M. (1996). Accidents and acts of God: A history of the terms. American Journal of Public Health, 86, 101-107. https://doi.org/10.2105/ajph.86.1.101

Lopes, L. L. (1982). Doing the impossible: A note on induction and the experience of randomness. Journal of Experimental Psychology: Learning, Memory, and Cognition, 8, 626-636. https://doi.org/10. 1037/0278-7393.8.6.626

Ma, H. (2002). Competitive advantage: What's luck got to do with it? Management Decision, 40, 525-536. https://doi.org/10.1108/ 00251740210433927

McDonald, S. (2010). Right place, right time: Serendipity and informal job matching. Socio-Economic Review, 8, 307-331. https://doi.org/ 10.1093/ser/mwp021

Mill, J. S. (1856). A system of logic. London, England: Parker.

Mlodinow, L. (2008). The drunkard's walk: How randomness rules our lives. Harmondsworth, England: Penguin books.

Neuringer, A. (1986). Can people behave "randomly?": The role of feedback. Journal of Experimental Psychology: General, 115, 62-75. https://doi.org/10.1037/0096-3445.115.1.62

Nickerson, R. (2002). The production and perception of randomness. Psychological Review, 109, 330-357. https://doi.org/10.1037/ 0033-295x.109.2.330

Nisbett, R., \& Ross, L. (1980). Human inference: Strategies and shortcomings of social judgment. Englewood Cliffs, NJ: Prentice-Hall.

Oskarsson, A. T., Van Boven L., McClelland, G. H., \& Hastie, R. (2009). What's next? Judging sequences of binary events. Psychological Bulletin, 135, 262-285. https://doi.org/10.1037/a0014821

Parnell, J. A., \& Dent, E. B. (2009). The role of luck in the strategyperformance relationship. Management Decision, 47, 1000-1021. https://doi.org/10.2139/ssrn.2331233

Reich, T., Kupor, D. M., \& Smith, R. K. (2018). Made by mistake: when mistakes increase product preference. Journal of Consumer Research, 44(5), 1085-1103. https://doi.org/10.1093/jcr/ucx089

Rescher, N. (1995). Luck: The brilliant randomness of everyday life. New York, NY: Farrar, Straus and Giroux.

Roberts, R. M. (1989). Serendipity: Accidental discoveries in science. New York, NY: Wiley.

Roese, N. J., \& Vohs, K. D. (2012). Hindsight bias. Perspectives on Psychological Science, 7, 411-426. 
Rogers, P., Qualter, P., \& Woods, D. (2016). The impact of event vividness, event severity, and prior paranormal belief on attributions towards a depicted remarkable coincidence experience: Two studies examining the misattribution hypothesis. British Journal of Psychology, 107(4), 710-751. https://doi.org/10.1111/bjop.12173

Rosset, E. (2008). It's no accident: Our bias for intentional explanations. Cognition 108, 771-780. https://doi.org/10.1016/j.cognition.2008. 07.001

Schreiber, J. M. (2014). Cognitive processes associated with the perception of randomness. Journal of Educational and Developmental Psychology, 4(1), 84-104. https://doi.org/10.5539/jedp.v4n1p84

Shanahan, M. J., \& Porfeli, E. J. (2006). Chance events in the life course. Advances in Life Course Research, 11, 97-119. https://doi.org/10. 1016/S1040-2608(06)11004-7

Sunstein, C. R. (2015). How Star Wars illuminates constitutional law. Michigan Law Review. Retrieved from http://nrs.harvard.edu/urn3:HUL.InstRepos:16148344

Taleb, N. N. (2005). Fooled by randomness: The hidden role of chance in life and in the markets. New York, NY: Random House.

Taleb, N. N. (2007). The black swan: The impact of the highly improbable. New York, NY: Random House.

Teigen, K. H., \& Jensen, T. K. (2011). Unlucky victims or lucky survivors: Spontaneous counterfactual thinking by families exposed to the tsunami disaster. European Psychologist, 16, 48-57. https://doi. org/10.1027/1016-9040/a000033
Teigen, K. H., \& Kanten, A. B. (2019). Are random events expected to be small? Psychological Research. https://doi.org/10.1007/s00426019-01252-9

Teigen, K. H., \& Keren, G. (2007). Waiting for the bus: When base rates refuse to be neglected. Cognition, 103, 337-357. https://doi.org/10. 1016/j.cognition.2006.03.007

Tyszka, T., Zielonka, P., Dacey, R., \& Sawicki, P. (2008). Perception of randomness and predicting uncertain events. Thinking \& Reasoning, 14(1), 83-110. https://doi.org/10.1080/13546780701677669

Wagenaar, W. A. (1988). Paradoxes of gambling behavior. Hove, England: Erlbaum.

Wagenaar, W. A., \& Keren, G. B. (1988). Chance and luck are not the same. Journal of Behavioral Decision Making, 1, 65-75. https://doi. org/10.1002/bdm.3960010202

Zhao, J., Hahn, U., \& Osherson, D. (2014). Perception and identification of random events. Journal of Experimental Psychology: Human Perception and Performance, 40, 1358-1371. https://doi.org/10. 1037/a0036816

Zhou, R., \& Pham, M. T. (2004). Promotion and prevention across mental accounts: When financial products dictate consumers' investment goal. Journal of Consumer Research, 31(1), 125-135. https://doi. org/10.1086/383429

Publisher's note Springer Nature remains neutral with regard to jurisdictional claims in published maps and institutional affiliations. 